\title{
Влияние ловушек в диоксиде кремния на пробой МОП-структур
}

\author{
(C) О.В. Александров \\ Санкт-Петербургский государственный электротехнический университет „ЛЭТИ“, \\ 197376 Санкт-Петербург, Россия \\ E-mail: Aleksandr_ov@mail.ru
}

(Получена 22 ноября 2016 г. Принята к печати 1 февраля 2017 г.)

\begin{abstract}
Разработана модель численного расчёта напряжения и времени задержки пробоя МОП-структур на основе механизма анодной дырочной инжекции, учитывающая распределение дырочных и электронных ловушек по толщине диэлектрика. Показано, что напряжение пробоя определяется накоплением заряда на дырочных ловушках в подзатворном диэлектрике вблизи катода, но зависит также от наличия дырочных ловушек вблизи анода и электронных ловушек. Время задержки пробоя в широком диапазоне напряженностей поля подчиняется экспоненциальной $1 / E$ зависимости и удовлетворительно описывает экспериментальные данные. При малых длительностях воздействия $\left(t<10^{-5} \mathrm{c}\right)$ пробой определяется накоплением заряда на свободных дырках.
\end{abstract}

DOI: $10.21883 /$ FTP.2017.08.44798.8457

\section{1. Введение}

Надежность МОП-интегральных схем определяется в значительной степени диэлектрической прочностью подзатворного диэлектрика, которая, в свою очередь, зависит от величины напряженности электрического поля и длительности приложения смещения. Различают несколько механизмов пробоя диоксида кремния в МОП-структурах. Принято считать, что при высоких напряженностях электрического поля $(E>8-10 \mathrm{MB} / \mathrm{cm})$ пробой происходит вследствие ударной ионизации в объеме диэлектрика — так называемый собственный пробой [1-3]. При меньших полях $(E \approx 6-8 \mathrm{MB} / \mathrm{cм})$ пробой может происходить по механизму анодной дырочной инжекции (АДИ-механизм) [4-6], когда энергии электронов достаточно для ударной ионизации материала анода. Образующиеся при этом дырки под действием электрического поля инжектируются из анода в диэлектрик, в котором захватываются на ловушки, изменяя электрическое поле. При низких полях имеет место т. н. несобственный пробой, обусловленный грубыми дефектами или загрязнениями диэлектрика [2,3]. Во всех случаях пробой инициируется электронами, инжектируемыми из катода путем либо прямого туннелирования при малых толщинах диэлектрика $(d<5$ нм), либо туннелирования по механизму Фаулера-Нортгейма - при бо́льших толщинах [7]. Необратимый пробой с нарушением целостности структуры происходит при некоторой критической величине инжектированного заряда, зависящей от напряженности поля и толщины диэлектрика [8]. При пробое величина инжектированного заряда дырок, определяемая по току подложки транзисторных МОП-структур, практически не зависит от напряженности электрического поля и толщины диэлектрика, что свидетельствует в пользу АДИ-механизма пробоя $[5,8]$. Накопление положительного заряда на дырочных ловушках приводит к увеличению напряженности электрического поля вблизи катода, что приводит к увеличению тока инжекции и усилению ударной ионизации в диэлектрике или материале анода. Возникающая таким образом положительная обратная связь приводит к резкому увеличению тока через структуру и локальному тепловому пробою в наиболее слабом дефектном месте диэлектрической пленки. Напряжение пробоя и время его задержки должны зависеть от концентрации и распределения дырочных и электронных ловушек в объеме диэлектрика МОП-структуры. В работе [9] была предложена количественная модель пробоя на основе генерации дырок путем ударной ионизации в объеме диоксида кремния. Этот механизм генерации требует высокой кинетической энергии носителей заряда ( 9эВ) и поэтому представляется менее вероятным, чем АДИ-механизм, для которого достаточно энергии электронов $\sim 5$ эВ [10].

В настоящей работе разработана модель численного расчета напряжения пробоя подзатворного диоксида кремния (ПЗО) на основе АДИ-механизма, учитывающая распределение дырочных и электронных ловушек по толщине диэлектрика.

\section{2. Уравнения модели}

При приложении отрицательного напряжения к затвору МОП-структуры через ПЗО начинает протекать электронный ток туннелирования по механизму Фаулера-Нортгейма [7]:

$$
I_{n}=A E_{c}^{2} \exp \left(-B / E_{c}\right),
$$

где $E_{c}-$ напряженность электрического поля вблизи затвора (катода), $A$ и $B-$ постоянные, определяемые эффективной массой электрона в диоксиде кремния и высотой потенциального барьера $\mathrm{SiO}_{2}$-затвор $\left(m_{\mathrm{ox}}=0.5 m_{e}, \phi=3.2\right.$ эВ для $n^{+}$-поликристаллического затвора [11]). Инжектированные из катода электроны ускоряются в электрическом поле диэлектрика и приобретают энергию, достаточную для ударной ионизации в кремниевой подложке (аноде). Часть образующихся 
при этом дырок преодолевает потенциальный барьер на границе $\mathrm{Si}-\mathrm{SiO}_{2}$ и под действием поля инжектируется обратно в диэлектрик. Величина дырочного тока определяется экспериментально по току подложки транзисторных МОП-структур $[8,12]$. Отношение дырочного тока $I_{p}$ к электронному $\alpha_{p}$ зависит от напряженности электрического поля вблизи анода $E_{a}$ и от толщины ПЗО [12]. Зависимость $\alpha_{p}\left(E_{a}\right)$ аппроксимировалась по данным работы [12] экспоненциальной $1 / E$ зависимостью:

$$
\alpha_{p}\left(E_{a}\right)=I_{p} / I_{n}=\alpha_{0} \exp \left(-B_{p} / E_{a}\right) .
$$

Инжектированные из анода дырки движутся к катоду, захватываются на дырочные ловушки и образуют положительный объемный заряд. Последний приводит к увеличению напряженности электрического поля вблизи катода, усилению инжекции электронов из катода, усилению инжекции дырок из анода, увеличению положительного объемного заряда и дальнейшему увеличению напряженности электрического поля вблизи катода. Эта положительная обратная связь приводит к резкому увеличению тока через ПЗО и, в конечном счете, - к пробою МОП-структуры в наиболее слабом месте.

Помимо захвата дырок $h^{+}$на нейтральные дырочные ловушки $T^{0}$ с образованием положительно заряженных центров $T^{+}$:

$$
T^{0}+h^{+} \stackrel{k_{1}}{\longrightarrow} T^{+},
$$

возможна нейтрализация последних при захвате электронов $e^{-}$:

$$
T^{+}+e^{-\stackrel{k_{2}}{\longrightarrow}} T^{0} \text {. }
$$

Учтем также захват электронов на нейтральные электронные ловушки $S^{0}$ с образованием отрицательно заряженных центров $S^{-}$:

$$
S^{0}+e^{-\stackrel{k_{3}}{\longrightarrow}} S^{-}
$$

и их нейтрализацию при захвате дырок:

$$
S^{-}+h^{+} \stackrel{k_{4}}{\longrightarrow} S^{0} \text {. }
$$

Процесс накопления зарядов и пробой ПЗО описываем следующими диффузионно-дрейфовыми уравнениями непрерывности и уравнением Пуассона:

$$
\begin{gathered}
\frac{\partial p}{\partial t}=D_{p} \frac{\partial^{2} p}{\partial x^{2}}-\mu_{p} \frac{\partial}{\partial x}(p E)-k_{1} C_{T}^{0} p-k_{4} C_{S}^{-} p \\
\frac{\partial n}{\partial t}=D_{n} \frac{\partial^{2} n}{\partial x^{2}}-\mu_{n} \frac{\partial}{\partial x}(n E)-k_{2} C_{T}^{+} n-k_{3} C_{S}^{0} n \\
\frac{\partial C_{T}^{+}}{\partial t}=-\frac{\partial C_{T}^{0}}{\partial t}=k_{1} C_{T}^{0} p-k_{2} C_{T}^{+} n \\
\frac{\partial C_{S}^{-}}{\partial t}=-\frac{\partial C_{S}^{0}}{\partial t}=k_{3} C_{S}^{0} n-k_{4} C_{S}^{-} p \\
\frac{\partial^{2} V}{\partial x^{2}}=-\frac{q}{\varepsilon \varepsilon_{0}}\left(C_{T}^{+}+p-C_{S}^{-}-n\right)
\end{gathered}
$$

где $x$ - координата, отсчитываемая от границы диоксида с кремнием $(x=0)$ до границы с затвором $(x=d) ; t$ - длительность приложения смещения; $n$ и $p$ - концентрации свободных электронов и дырок в $\mathrm{SiO}_{2}$, соответственно; $D_{n}$ и $D_{p}-$ коэффициенты диффузии электронов и дырок в $\mathrm{SiO}_{2}$ соответственно $\left(D_{n}=0.5 \mathrm{~cm}^{2} / \mathrm{c}, D_{p}=1 \cdot 10^{-7} \mathrm{~cm}^{2} / \mathrm{c}[13]\right) ; \mu_{n}$ и $\mu_{p}-$ подвижности электронов и дырок соответственно; $C_{T}^{0}$ и $C_{T}^{+}$- концентрации нейтральных и положительно заряженных дырочных ловушек; $C_{S}^{0}$ и $C_{S}^{-}-$концентрации нейтральных и отрицательно заряженных электронных ловушек; $V$ - распределение потенциала в диоксиде кремния; $E$ - напряженность электрического поля в диэлектрике; $E=-d V / d x ; q-$ заряд электрона; $\varepsilon-$ относительная диэлектрическая проницаемость диоксида кремния $(\varepsilon=3.9) ; \varepsilon_{0}$ - диэлектрическая постоянная.

Уравнения (7)-(11) решались при следующих граничных условиях. Поток электронов из катода определяется током туннелирования Фаулера-Нортгейма (1) при напряженности электрического поля вблизи катода при $x=d(d-$ толщина ПЗО):

$$
j_{n}(d, t)=I_{n}\left(E_{c}\right) / q .
$$

Поток дырок из анода (2) определяется при напряженности электрического поля вблизи анода при $x=0$ :

$$
j_{p}(0, t)=\alpha_{p}\left(E_{a}\right) I_{n}\left(E_{c}\right) / q .
$$

Граница диэлектрика с анодом полагается поглощающей для электронов, а граница диэлектрика с катодом поглощающей для дырок:

$$
n(0, t)=p(d, t)=0 \text {. }
$$

К затвору относительно подложки приложено напряжение $V_{g}$ :

$$
V(0, t)=0, \quad V(d, t)=V_{g}
$$

Полагаем, что в начальный момент времени концентрации всех компонентов нулевые:

$$
n(x, 0)=p(x, 0)=C_{T}^{+}(x, 0)=C_{S}^{-}(x, 0)=0,
$$

кроме исходной концентраций нейтральных дырочных и электронных ловушек. Как показано в работах [14,15], дырочные ловушки располагаются вблизи межфазных границ, и их концентрация спадает экспоненциально вглубь диэлектрика:

$$
C_{T 1}^{0}(x)=C_{T 01} \exp \left(-x / L_{1}\right)=\left(Q_{T 1}^{0} / L_{1}\right) \cdot \exp \left(-x / L_{1}\right),
$$

$C_{T 2}^{0}(x)=C_{T 02} \exp \left[(x-d) / L_{2}\right]=\left(Q_{T 2}^{0} / L_{2}\right) \cdot \exp \left[(x-d) / L_{2}\right]$,

где $Q_{T 1}^{0}$ и $Q_{T 2}^{0}-$ полные концентрации нейтральных дырочных ловушек у анода и катода соответственно, $L_{1}$ и $L_{2}$ - ширина распределения дырочных ловушек у анода и катода соответственно, полагаем 
$L_{1}=L_{2}=L=1.2$ нм [15]. Распределение электронных ловушек по толщине как правило однородно [16]:

$$
C_{S}^{0}(x)=C_{S 0}^{0}=Q_{S}^{0} / d,
$$

где $Q_{S}^{0}$ - полная концентрация нейтральных электронных ловушек в ПЗО.

Константы скоростей реакций (3)-(6) при диффузионном ограничении определяются коэффициентами диффузии подвижных компонентов: $k_{1}=\sigma_{p}^{0} V_{\mathrm{th}} D_{p} / D_{n}$, $k_{2}=\sigma_{n}^{+} V_{\mathrm{th}} ; \quad k_{3}=\sigma_{n}^{0} V_{\mathrm{th}} ; \quad k_{4}=\sigma_{p}^{-} V_{\mathrm{th}} D_{p} / D_{n}$, где $\sigma_{p}^{0} \quad$ и $\sigma_{p}^{-}$- сечения захвата дырок на нейтральные и отрицательно заряженные ловушки, $\sigma_{n}^{0}$ и $\sigma_{n}^{+}-$сечения захвата электронов на нейтральные и положительно заряженные ловушки соответственно, $V_{\text {th }}-$ тепловая скорость электронов $\left(V_{\text {th }} \cong 10^{7} \mathrm{~cm} / \mathrm{c}\right)$. Учитывалась зависимость сечений захвата от напряженности электрического поля для дырок $[17,18]$ :

$$
\begin{gathered}
\sigma_{p}^{0}(E)=\sigma_{p 0}^{0}\left(1+1.9 \cdot 10^{-4} E^{0.55}\right)^{-1}, \\
\sigma_{p}^{-}(E)=\sigma_{p 0}^{-} \quad \text { при } \quad E \leq 3 \cdot 10^{5} \mathrm{~B} / \mathrm{cm}, \\
\sigma_{p}^{-}(E)=6.0 \cdot 10^{-10} E^{-0.6} \quad \text { при } \quad E>3 \cdot 10^{5} \mathrm{~B} / \mathrm{cm},
\end{gathered}
$$

и для электронов $[16,19,20]$ :

$$
\sigma_{n}^{0}(E)=\sigma_{n 0}^{0},
$$

$\sigma_{n}^{+}(E)=\sigma_{n 0}^{+}\left(1+8.3 \cdot 10^{-9} E^{1.5}\right)^{-1}$ при $\quad E \leq 7 \cdot 10^{5} \mathrm{~B} / \mathrm{cm}$,

$\sigma_{n}^{+}(E)=\sigma_{n 0}^{+}\left(1+8.7 \cdot 10^{-17} E^{2.865}\right)^{-1}$ при $E>7 \cdot 10^{5} \mathrm{~B} / \mathrm{cm}$,

где $\sigma_{p 0}^{0}, \sigma_{p 0}^{-}, \quad \sigma_{n 0}^{+}-$сечения захвата дырок и электронов соответственно в слабых полях $\left(\sigma_{p 0}^{0}=1.4 \cdot 10^{-14} \mathrm{~cm}^{2}, \quad \sigma_{p 0}^{-}=3.1 \cdot 10^{-13} \mathrm{~cm}^{2}, \quad \sigma_{n 0}^{0}=\right.$ $\left.=1 \cdot 10^{-17} \mathrm{~cm}^{2}, \quad \sigma_{n 0}^{+}=1.6 \cdot 10^{-12} \mathrm{~cm}^{2} \quad[16-20]\right)$. Зависимость (22b) определялась в работе [20] до величины поля $3 \cdot 10^{6} \mathrm{~B} / \mathrm{cm}$. При более высоких полях вблизи пробоя падение сечения захвата электронов с полем становится, по-видимому, более сильным (см. ниже), поэтому полагали

$$
\sigma_{n}^{+}(E)=0 \text { при } E>5 \cdot 10^{6} \mathrm{~B} / \mathrm{cm} .
$$

\section{3. Расчеты по модели и обсуждение}

Система уравнений (7)-(11) с граничными условиями (12)-(15), начальными условиями (16)-(18), с учетом условий $(19)-(22 \mathrm{c})$ решались численно с использованием неявной разностной схемы.

Зависимости туннельного электронного потока от времени при различных напряжениях на затворе показаны на рис. 1. Как видно из рисунка, при некотором времени выдержки МОП-структуры под напряжением происходит резкое увеличение тока, переходящее в пробой. За время задержки пробоя $t_{\mathrm{BD}}$ принимаем время, при

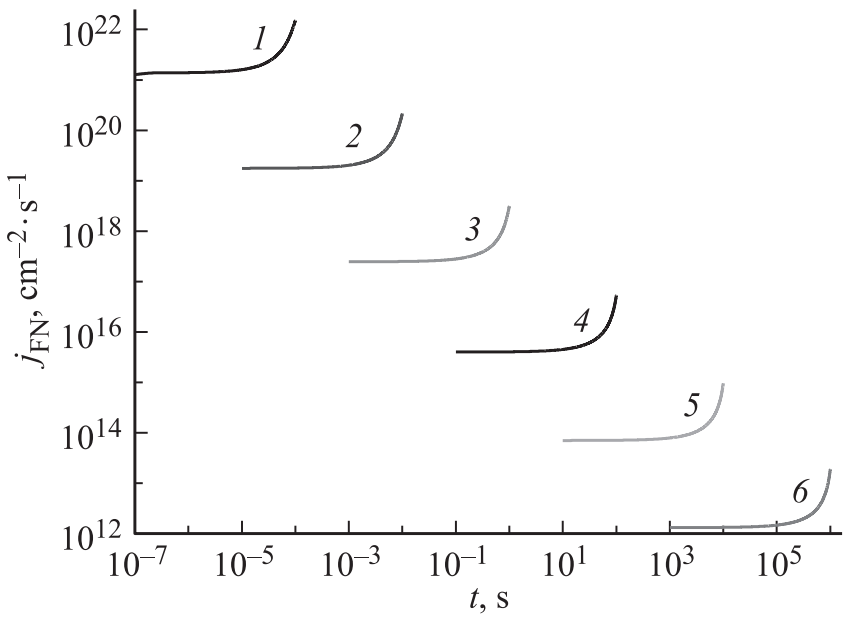

Рис. 1. Зависимость туннельного электронного потока от времени при напряжении на затворе, В: $1-19.4,2-15.4$, $3-12.7,4-10.8,5-9.4,6-8.4\left(Q_{T 2}^{0}=1 \cdot 10^{13} \mathrm{~cm}^{-2}\right.$, $d=7.9 \mathrm{HM})$.

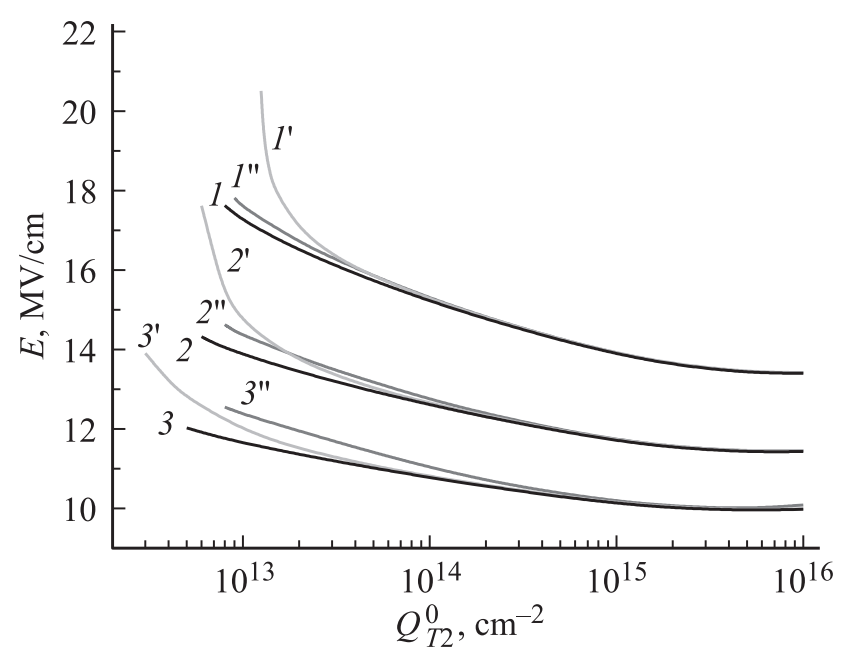

Рис. 2. Зависимости пробивной напряженности поля от $Q_{T 2}^{0}$ при $Q_{T 1}^{0}, \mathrm{~cm}^{-2}: 1,2,3,1^{\prime \prime}, 2^{\prime \prime}, 3^{\prime \prime}-0 ; 1^{\prime}, 2^{\prime}, 3^{\prime}-10^{14}$ и при $Q_{S}^{0}, \mathrm{~cm}^{-2}: 1,2,3,1^{\prime}, 2^{\prime}, 3^{\prime}-0 ; 1^{\prime \prime}, 2^{\prime \prime}, 3^{\prime \prime}-10^{14}$. Время, с: 1 , $1^{\prime}, 1^{\prime \prime}-10^{-3} ; 2,2^{\prime}, 2^{\prime \prime}-10^{-1} ; 3,3^{\prime}, 3^{\prime \prime}-10$.

котором электронный ток инжекции увеличивается по сравнению с начальным значением на порядок. Напряжение пробоя $V_{\mathrm{BD}}$ и соответствующая ему напряженность электрического поля $E_{\mathrm{BD}}$ зависят от полной концентрации дырочных и электронных ловушек (рис. 2). Дырочные ловушки вблизи катода $Q_{T 2}$ уменьшают пробивную напряженность (рис. 2, кривые $1-3$ ). Дырочные ловушки вблизи анода оказывают компенсирующее влияние на пробой при $Q_{T 1}^{0}>Q_{T 2}^{0}$ (рис. 2, кривые $\left.l^{\prime}-3^{\prime}\right)$. При этом ширина распределения ловушек $L$ не оказывала заметного влияния на напряжение пробоя. Однородно распределенные электронные ловушки также оказывают компенсирующее влияние на пробой при $Q_{S}^{0}>Q_{T 2}^{0}$ (рис. 2, кривые $1^{\prime \prime}-3^{\prime \prime}$ ). 


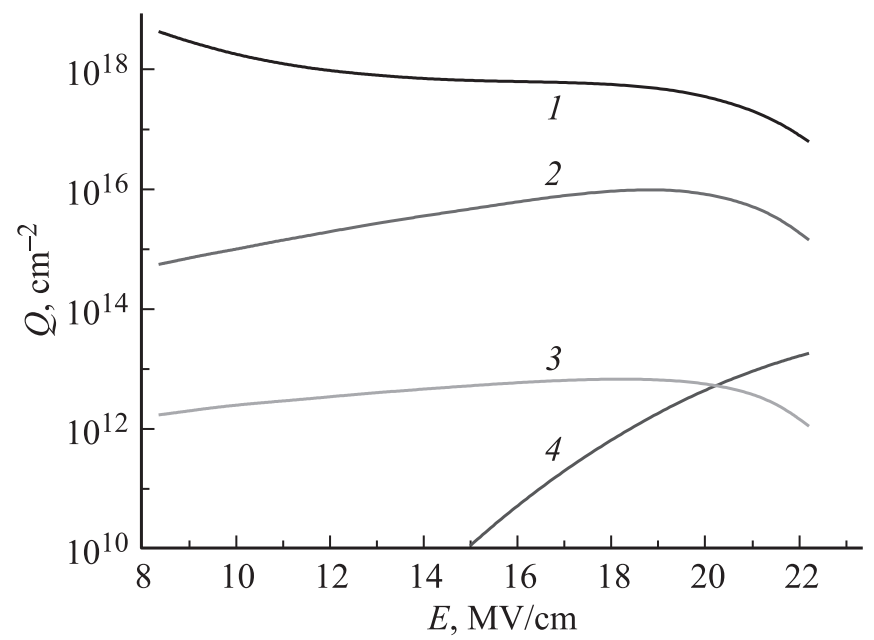

Рис. 3. Зависимость $Q_{n}^{\mathrm{inj}}(1), Q_{p}^{\mathrm{inj}}(2), Q_{T}^{+}$(3) и $Q_{h}^{+}$(4) от напряженности поля при пробое $\left(Q_{T 2}^{0}=6 \cdot 10^{12} \mathrm{~cm}^{-2}, Q_{T 1}^{0}=0\right.$, $\left.Q_{S}^{0}=0\right)$.

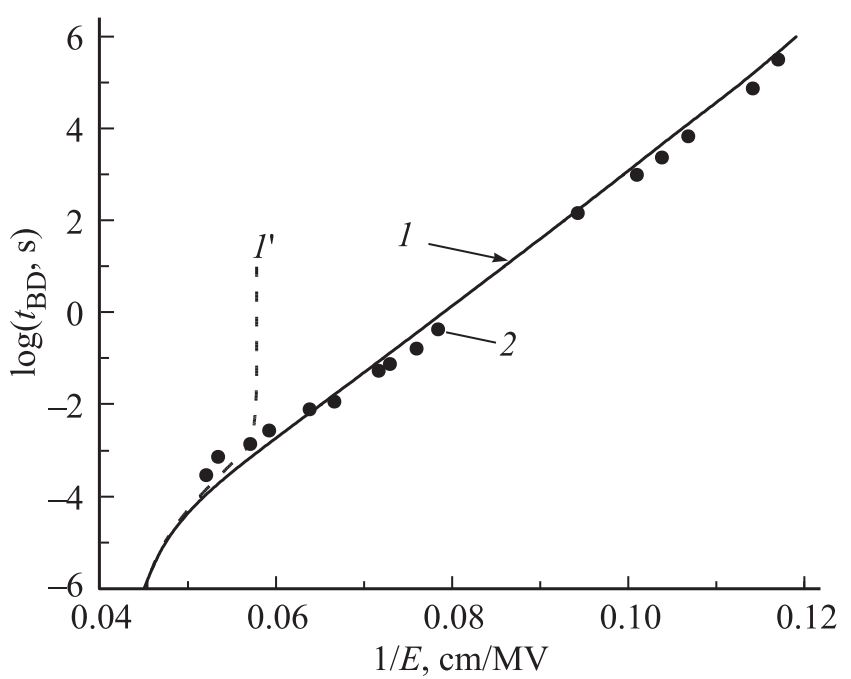

Pис. 4. Зависимость времени задержки пробоя от напряженности поля: $1,1^{\prime}-$ расчет при $Q_{T 2}^{0}=1 \cdot 10^{13} \mathrm{~cm}^{-2}, Q_{T 1}^{0}=0$, $Q_{S}^{0}=0 ; 2-$ эксперимент [9].

При пробое полный заряд электронов, инжектированных из катода, $\left(Q_{n}^{\mathrm{inj}}\right)$, с увеличением напряженности электрического поля уменьшается (рис. 3, кривая 1), a инжектированный из анода полный заряд дырок $Q_{p}^{\text {inj }}$ и положительный объемный заряд на дырочных ловушках $Q_{T}^{+}$относительно слабо растут (рис. 3, кривые 2,3$)$ до напряженности поля $E \approx 20 \mathrm{MB} / \mathrm{cm}$. При $E>20 \mathrm{MB} / \mathrm{cm}$ и малых временах $\left(t<10^{-4} \mathrm{c}\right)$ полный заряд свободных дырок $Q_{h}^{+}$превышает положительный объемный заряд на дырочных ловушках $Q_{T}^{+}$(рис. 3, кривая 4).

Время задержки пробоя с увеличением напряженности электрического поля уменьшается (рис. 4, кривая 1) в соответствии с экспоненциальной $1 / E$ за- висимостью $\left(t_{\mathrm{BD}}=t_{0} \exp (C / E)\right)$ в широком диапазоне напряженностей поля (от 8 до $20 \mathrm{MB} / \mathrm{cm}$ ). Отклонение от экспоненциальной $1 / E$ зависимости при малых временах $\left(t<10^{-5} \mathrm{c}\right)$ и высоких напряженностях поля связано с конечным временем захвата дырок на ловушки $\left(\tau_{P}=1 /\left(k_{1} C_{T 02}\right) \approx 10^{-6}\right.$ с при $\left.Q_{T 2}^{0}=1 \cdot 10^{13} \mathrm{~cm}^{-2}\right)$. Пробой в этом случае происходит вследствие накопления положительного заряда на свободных дырках (рис. 3, кривая 4). Расчеты по модели сравнивались с экспериментальными данными [9] по времени задержки пробоя для МОП-структур с поликристаллическим затвором и толщиной термического ПЗО $d=7.9$ нм (рис. 4, точки 2). Соответствие расчета с экспериментом достигается при полной концентрации дырочных ловушек вблизи катода $Q_{T 2}^{0}=1 \cdot 10^{13} \mathrm{~cm}^{-2}$. Найденная концентрация дырочных ловушек по величине соответствует диапазону литературных данных для МОП-структур с поликристаллическим затвором $[15,16]$.

Пунктиром (рис. 4, кривая $I^{\prime}$ ) показан расчет при $\sigma_{n}^{+}(E)$ по по формуле $(22 \mathrm{~b})$. В этом случае при достаточно больших временах устанавливается равновесие между захватом дырок по реакции (3) и их рекомбинации с электронами по реакции (4), в результате чего пробивная напряженность поля перестает зависеть от времени. Видно, что кривая $l^{\prime}$ (рис. 4) не соответствует экспоненциальной $1 / E$ и экспериментальной зависимостям, то есть равновесие в реакциях (3) и (4) не достигается. Этот результат можно объяснить более сильным падением $\sigma_{n}^{+}(E)$ при высоких полях, чем по формуле (22b).

\section{4. Заключение}

Таким образом, на основе АДИ-механизма разработана модель численного расчета напряжения пробоя и времени задержки пробоя МОП-структур. Напряжение пробоя МОП-структур определяется полной концентрацией дырочных и электронных ловушек в подзатворном диэлектрике, при этом ширина распределения не оказывает заметного влияния. Дырочные ловушки вблизи анода и равномерно распределенные электронные ловушки оказывают компенсирующее влияние на пробой при $Q_{T 1}^{0}$ и $Q_{S}^{0}>Q_{T 2}^{0}$. При пробое положительный инжектированный заряд $Q_{p}^{\text {inj }}$ и объемный заряд, накапливающийся на дырочных ловушках вблизи катода $Q_{T 2}^{+}$, относительно слабо зависят от напряженности электрического поля. Время задержки пробоя в широком диапазоне напряженностей поля (от 8 до $20 \mathrm{MB} / \mathrm{cm}$ ) подчиняется экспоненциальной $1 / E$ зависимости. Расчет по модели удовлетворительно описывает экспериментальные данные [9] при полной концентрации дырочных ловушек вблизи катода $Q_{T 2}^{0}=1 \cdot 10^{13} \mathrm{~cm}^{-2}$. При малых длительностях воздействия $\left(t<10^{-5} \mathrm{c}\right)$ пробой определяется накоплением заряда на свободных дырках. 


\section{Список литературы}

[1] T.H. DiStefano, M. Shatzkes. Appl. Phys. Lett., 25, 685 (1974).

[2] P. Solomon. J. Vac. Sci. Technol., 14, 1122 (1975).

[3] N. Klein. Thin Sol. Films, 50, 223 (1978).

[4] M.V. Fischetti. Phys. Rev. B, 31, 2099 (1985).

[5] K.F. Schuegraf, C. Hu. J. Appl. Phys., 76, 3695, 1994.

[6] P. Samanta. Appl. Phys. Lett., 75, 2966 (1999).

[7] M. Lenzlinger, E.H. Snow. J. Appl. Phys., 40, 278 (1969).

[8] C. Chen, S.E. Holland, K.K. Yong, C. Chang, C. Hu. Appl. Phys. Lett., 49, 669 (1986).

[9] C. Chen, S.E. Holland, C. Hu. IEEE Trans. Electron. Dev., 32, 413 (1985).

[10] D.J. DiMaria, E. Cartier, D.A. Buchanan. J. Appl. Phys., 80, 304 (1996).

[11] P. Samanta, C.K. Sarkar. Sol. St. Electron., 46, 279 (2002).

[12] K. Kobayashi, A. Teramoto, M. Hirayama, Y. Fujita. J. Appl. Phys., 77, 3277 (1995).

[13] R.C. Huges. Phys. Rev. Lett., 30, 1333 (1973).

[14] V.K. Adamchuk, V.V. Afanas'ev. Progr. Surf. Sci., 41. 111 (1992).

[15] Q.D.M. Khosru, N. Yasuda, K. Taniguchi, C. Hamaguchi. J. Appl. Phys., 76, 4738 (1994).

[16] А.П. Барабан, В.В. Булавинов, П.П. Коноров. Электроника слоев $\mathrm{SiO}_{2}$ на кремнии (Л., 1988).

[17] R.J. Krantz, L.W. Aukerman, T.C. Zietlow. IEEE Trans. Nucl. Sci., 34, 1196 (1987).

[18] J.J. Tzou, J.Y.C. Sun, C.T. Sah. Appl. Phys. Lett., 43, 8611 (1983).

[19] H.E. Boesch, F.B. McLean, J.M. Benedetto, J.M. McGarrity. IEEE Trans. Nucl. Sci., 33, 1191 (1986).

[20] T.H. Ning. J. Appl. Phys., 47, 3203 (1976).

Редактор А.Н. Смирнов

\section{Influence of traps in silcon dioxide on MOS-structure breakdown}

\section{O.V. Aleksandrov}

St. Petersburg State Electrotechnical University „LETI“, 197376 St. Petersburg, Russia

Abstract The model of numerical calculation of field and time of a delay of breakdown of MOS-structures on the basis of the mechanism of anode hole injection considering distribution of hole and electronic traps on dielectric thickness is developed. It is shown that breakdown field is defined by accumulation of a charge on hole traps in gate dielectric near the cathode, but depends also on existence of hole traps near the anode and electonic traps. Breakdown delay time in the wide tange of field submits to exponential $1 / E$ dependences and well describes experimental data. At small dutation of influence $\left(t<10^{-5} \mathrm{~s}\right)$ breakdown is defined by accumulation of a charge on free holes. 